\title{
Thermodynamic, kinetic and electronic structure aspects of a charge-transfer active bichromophoric organofullerene
}

\author{
K SENTHIL KUMAR and ARCHITA PATNAIK* \\ Department of Chemistry, Indian Institute of Technology Madras, Chennai 600 036, India \\ e-mail: archita59@yahoo.com
}

MS received 11 January 2012; revised 24 May 2012; accepted 4 October 2012

\begin{abstract}
Our recent work on charge transfer in the electronically push-pull dimethylaminoazobenzenefullerene $\mathrm{C}_{60}$ donor-bridge-acceptor dyad through orbital picture revealed charge displacement from the $\mathrm{n}_{(\mathrm{N}=\mathrm{N})}$ (non-bonding) and $\pi_{(\mathrm{N}=\mathrm{N})}$ type orbitals centred on the donor part to the purely fullerene centred LUMOs and $(\mathrm{LUMO}+\mathrm{n})$ orbitals, delocalized over the entire molecule. Consequently, this investigation centres around the kinetic and thermodynamic parameters involved in the solvent polarity dependent intramolecular photo-induced electron transfer processes in the dyad, indispensable for artificial photosynthetic systems. A quasi-reversible electron transfer pathway was elucidated with electrode-specific heterogeneous electron transfer rate constants.
\end{abstract}

Keywords. Donor-acceptor dyad; organo fullerene; intramolecular charge transfer; electronic structure calculation; solvent polarity; cyclic voltammetry.

\section{Introduction}

The alluring intramolecular electronic interactions in electronically asymmetric Donor-Bridge-Acceptor (dyad) systems based on $\mathrm{C}_{60}$ have been a major concern in molecular materials research. ${ }^{1,2}$ Distance, conjugation and the nature of donor/acceptor species affect the degree of interaction between the donor and the acceptor and can be controlled by incorporating a suitable linking group. ${ }^{3,4}$ Thus, systematic exploration of chemical and electronic nature of the linking group at varying conditions for a set of donor and acceptor moieties is important for accurate interpretation of molecular signatures obtained from spectroscopic methods towards a suitable device application. In line with the above, a recent work reported from our laboratory dealt with a $\mathrm{C}_{60}$-based dyad that showed excellent rectification with a rectification ratio of $154 .{ }^{5}$

Molecular components based on fullerenes offer a unique possibility of combining the advantageous three-dimensional framework with electron accepting character, enabled by the triply degenerate LUMO. ${ }^{6-9}$ On the other hand, azobenzenes with two different conformers, addressable by light or electric field were proven as building blocks for the construction of stimuli-responsive materials with innumerable applications, ranging from conformation switching of proteins

*For correspondence to data storage. ${ }^{10-14}$ Covalent tailoring of azobenzene and fullerene could provide novel stimuli-tunable materials with accessible redox states and lower excitation energies, desirable for the construction of optical data storage materials. A variety of fullerene-azobenzene hybrids were synthesized and studied ${ }^{15-17}$ among them, the molecular system investigated by Guldi et al. ${ }^{18}$ is the only report that dealt with electron transfer mediating ability of the pure azobenzene bridge. The above facets clearly emphasize the need for a prototype fullerene-azobenzene based molecular system along with the elucidation of various electron transfer parameters as reported for fullerene-porphyrin ${ }^{19-21}$ and fullerene-CNT ${ }^{22,23}$ based dyads. The reorganization energy $(\lambda)$, the free energy change $\left(\Delta \mathrm{G}_{\mathrm{CS}}\right)$ and the activation energy barrier $\left(\Delta \mathrm{G}_{\mathrm{CS}}^{++}\right)$for charge separation in a photo-induced electron transfer process are the guiding parameters for the selection of a molecular system towards a specific artificial photosynthetic application. ${ }^{24}$

In estimating the electron transfer parameters for fullerene-azobenzene hybrid dyadic systems, we designed a novel push-pull, structurally noncentrosymmetric and electronically asymmetric fullerene $N, N$-dimethylaminoazobenzene (DPNME) molecular skeleton (cf. figure 1) in which the electron donating $N, N$-dimethylaniline moiety intensified charge density on the $\beta$-azonitrogen. Our initial investigation revealed the existence of ground state intramolecular electronic interaction in DPNME. ${ }^{25}$ 
Apart from this, the dyadic system was found to show (i). frontier molecular orbital switching upon addition of a proton as an external chemical input stimuli, ${ }^{26}$ (ii). solvent polarity dependent specific intermolecular association into $\mathrm{J}$ and $\mathrm{H}$ aggregates with unique $2 \mathrm{D}$ organization in its crystal structure facilitated by inter and intra dimer fullerene-fullerene and fullereneazobenzene interactions ${ }^{27}$ and (iii) formation of multidimensional tunable supramolecular architectures at varying experimental conditions. ${ }^{28}$ The multifaceted nature of the DPNME dyadic system prompted further investigations to unravel the photo-induced intra molecular electronic interactions and the associated thermodynamic and kinetic parameters.

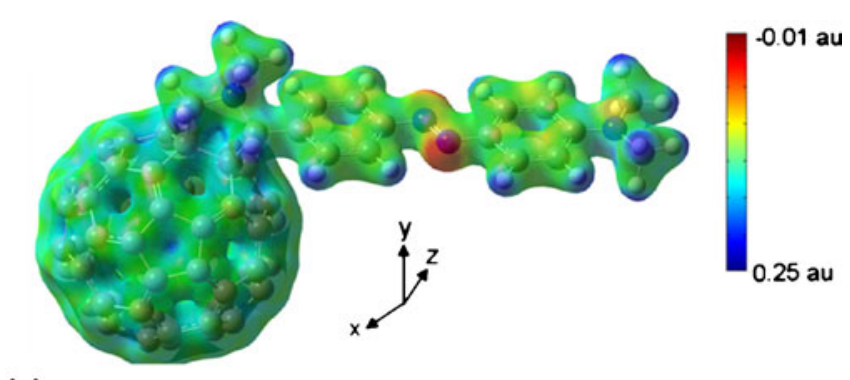

(a)

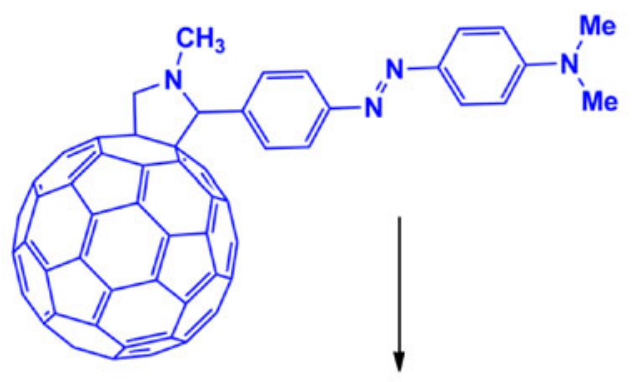

(b)

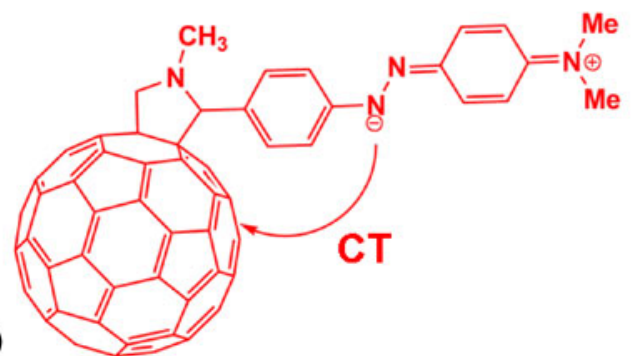

Figure 1. (a) Molecular electrostatic potential of the dyad mapped on the total electron density at the B3LYP/3-21g (d, p) level of theory indicating greater electron density on the azo nitrogens than anilinic nitrogens due to the resonance stabilization. (b) Molecular structures of (E)-N, N-dimethyl-4(p-pyrrolidinofullerenediazenyl) aniline (DPNME) showing through-bond representation of the charge transfer pathway operating in the dyad. ${ }^{25}$

\section{Experimental and computational methods}

Synthetic details of the DPNME dyad were reported in our previous report. ${ }^{25}$ Electronic absorption spectra were measured with a Schimadzu double beam UVVis spectrophotometer. Emission spectra of the samples were obtained from a Jobin-Yvon Fluorolog spectrofluorimeter with $0.2 \mathrm{~nm}$ resolution, with sample geometry at $90^{\circ}$ to the excitation source. Cyclic Voltammetry (CV) was carried out with a $\mathrm{CH}$ instrument's 660B electrochemical analyzer. Benzonitrile was used as the solvent and $0.1 \mathrm{M}$ Tetrabutylammonium hexafluorophosphate was used as the supporting electrolyte with a potential window ranging from $+3 \mathrm{~V}$ to $-3 \mathrm{~V}$. The Glassy Carbon/Pt electrode embedded in a Teflon rod (CHI $1.5 \mathrm{~mm}$ in diameter) was polished with $0.1 \mu \mathrm{m}$ $\gamma$-alumina powder to mirror finish and was used as the working electrode. $\mathrm{Ag} / \mathrm{AgNO}_{3}$ non-aqueous electrode was used as the reference. A pre-cleaned Pt wire was used as the counter electrode. The analyte concentration was fixed at $5 \times 10^{-4} \mathrm{M}$, unless otherwise stated. Dissolved oxygen was removed by purging with high pure nitrogen gas prior to experiments.

Density Functional calculations were carried out using Gaussian 03 set of programmes. ${ }^{29}$ The groundstate geometry of each molecule was fully optimized using the hybrid B3LYP functional with 6-31g (d, p) basis set. In B3LYP ${ }^{30}$ the exchange is a combination of $20 \% \mathrm{HF}$ exchange, Slater functional, and Becke's GGA correction, ${ }^{31}$ whereas the correlation part combines $\mathrm{VWN}^{32}$ and $\mathrm{LYP}^{33}$ functionals. TD-DFT methodology ${ }^{34,35}$ was then used to compute the excitation energies, oscillator strengths and the composition of electronic transitions. The bulk solvent effects were evaluated during the TD-DFT calculations by means of the standard Polarizable Continuum Model (IEFPCM). ${ }^{36,37}$ In PCM, the problem was divided into a solute part lying inside a cavity and a solvent part represented as a structureless material, characterized by its macroscopic properties (dielectric constant, radius, density and molecular volume).

\section{Results and discussion}

\subsection{Ground state electronic structure of the DPNME dyad}

The ground state electronic structure of trans- DPNME was obtained from DFT calculations in vacuum utilizing B3LYP level of theory with 6-31G (d, p) basis set. The geometry optimized structure and the frontier molecular orbitals are depicted in figure 2. With an estimated ground state dipole moment of $7.07 \mathrm{D}$, 

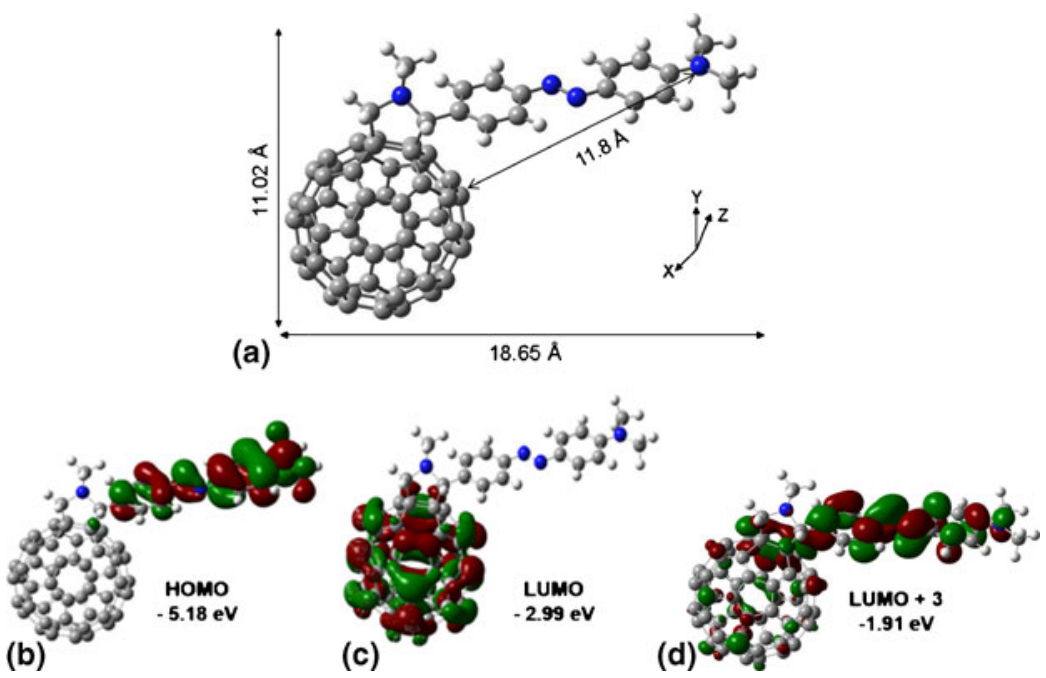

Figure 2. (a). B3LYP/ $6-31 \mathrm{G}(\mathrm{d}, \mathrm{p})$ full optimized geometry of DPNME. (b), (c) and (d) show the HOMO, LUMO and LUMO +3 molecular orbitals of DPNME. Distance between the anilinic nitrogen and nearest fullerene carbon is shown.

the dyad HOMO at $5.18 \mathrm{eV}$ was located on the $\mathrm{N}, \mathrm{N}-$ dimethylaniline donor, extending onto the azo bridge. The LUMO at $2.99 \mathrm{eV}$ was located on the $\mathrm{C}_{60}$ part of the dyad and the electronic band gap of the latter was estimated to be $2.12 \mathrm{eV}$.

The HOMO-LUMO picture clearly reveals the disjoint nature of the frontier molecular orbitals. This property was desirable in terms of device applications especially to envision the system as a unimolecular electronic rectifier as evidenced from previous reports. ${ }^{38,39}$ The parameter 'potential drop', which is the energy difference between the LUMO+n molecular orbital located on the donor and LUMO on the acceptor is an important entity in determining the current onset

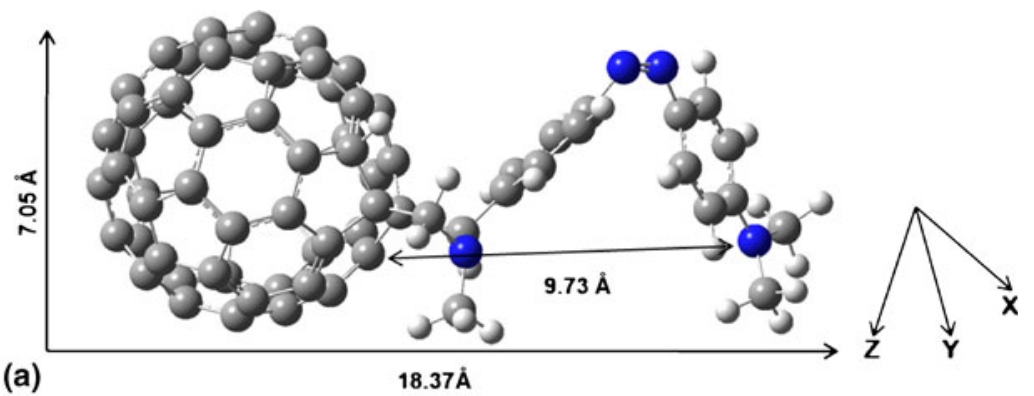

(b)

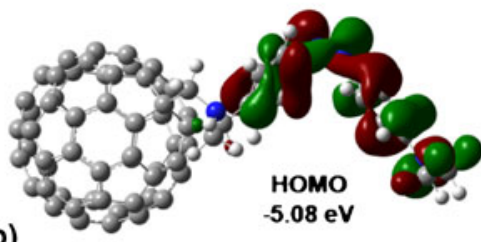

(d)

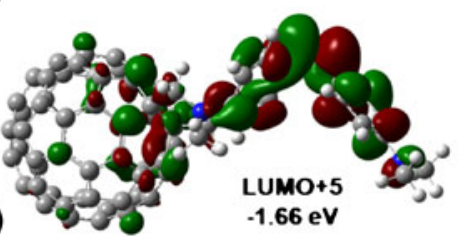

(c)

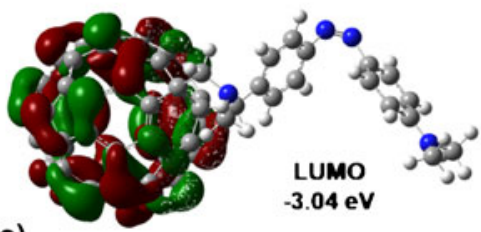

(e)

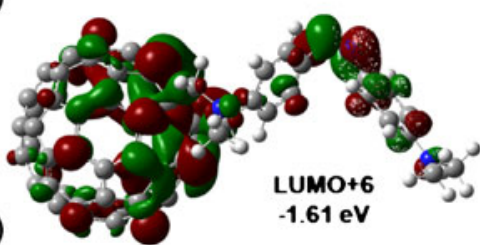

Figure 3. (a). B3LYP/ $6-31 \mathrm{G}(\mathrm{d}$, p) full optimized geometry of cisDPNME. (b), (c), (d) and (e) show the HOMO, LUMO, LUMO+5 and LUMO +6 molecular orbitals of cis-DPNME. Distance between the anilinic nitrogen and nearest fullerene carbon is shown. 
Table 1. A comparison of the trans (extended) and the cis (folded) conformations of the DPNME dyad.

\begin{tabular}{lcccc}
\hline $\begin{array}{l}\text { Dyad } \\
\text { conformation }\end{array}$ & $\begin{array}{c}\text { Ground state } \\
\text { dipole moment (D) }\end{array}$ & $\begin{array}{c}\text { Ground state } \\
\text { energy (a. u.) }\end{array}$ & $\begin{array}{c}\text { HOMO-LUMO } \\
\text { Gap (eV) }\end{array}$ & $\begin{array}{c}\Delta \mathrm{E}_{\text {LUMO }} \\
(\mathrm{eV})\end{array}$ \\
\hline trans-DPNME & 7.07 & -3165.0190 & 2.12 & 1.08 \\
cis-DPNME & 7.31 & -3164.9822 & 2.04 & 1.43 \\
\hline
\end{tabular}

in a molecular rectification process. Majumdar and coworkers reported potential drops for a range of donoracceptor systems based on DFT calculations. ${ }^{40}$ An exact correlation between the predicted potential drop and the experimental onset of current was reported in a rectification process based on a fullerene-dodecyloxy benzene dyad ${ }^{5}$ from our laboratory. A potential drop of $1.08 \mathrm{eV}$ for the present DPNME dyad reveals a possible low threshold voltage rectification of the system when placed appropriately between two electrodes.

In-order to probe the isomerization-induced structural changes and the associated frontier molecular orbital alignment, DFT calculation on the cis-DPNME dyad was carried out as depicted in figure 3. The comparatively higher calculated dipole moment of 7.31 D and a reduced HOMO-LUMO gap of $2.04 \mathrm{eV}$ for the cis-DPNME dyad (cf. table 1) implied more favourable electronic interactions in the cis-DPNME dyad upon isomerization, exemplified from the much reduced edge-to-edge distance between the donor aniline $\mathrm{N}$ and the acceptor $\mathrm{C}_{60}(9.73 \AA$ in cis vs. $11.8 \AA$ in trans dyad). The characteristic frontier molecular orbital localization further strengthened the single molecular device characteristic of the molecular skeleton. In addition, the delocalized nature of the LUMO +5 and LUMO +6 orbitals associated with the ${ }^{1}\left(\mathrm{n} \rightarrow \pi^{*}\right)$ and ${ }^{1}(\pi \rightarrow \pi)^{*}$ electronic transitions respectively revealed mixing of charge transfer character with the ${ }^{1}\left(\mathrm{n} \rightarrow \pi^{*}\right)$ and ${ }^{1}(\pi \rightarrow \pi)^{*}$ transitions, as was observed for the trans-DPNME dyad, discussed in the following section.

\subsection{Ground state charge transfer in trans-DPNME dyad in varying solvent polarity}

Electronic absorption spectra of the dyad in solvents of varying polarity in figure 4 a depict the ${ }^{1}\left(n \rightarrow \pi^{*}\right)$ transition located at $\sim 447 \mathrm{~nm}$ in toluene, dichloromethane and a bathochromically shifted feature at $465 \mathrm{~nm}$ benzonitrile. The corresponding ${ }^{1}\left(\pi \rightarrow \pi^{*}\right)$ features in the dyad were observed at $414 \mathrm{~nm}$ in toluene with a $5 \mathrm{~nm}$ red shift in benzonitrile. The electronic absorption located at $\sim 432 \mathrm{~nm}$ in all solvents was attributed to a fullerene $\mathrm{C}_{60}$ centred transition via the literature report. ${ }^{41}$ TD-DFT calculations performed on DPNME in solvents of varying polarity clearly reproduced the experimental trend as depicted in figure $4 \mathrm{~b}$. Due to the non-parameterization of benzonitrile in Gaussian 03 set of programs, dimethylsulphoxide was used as a
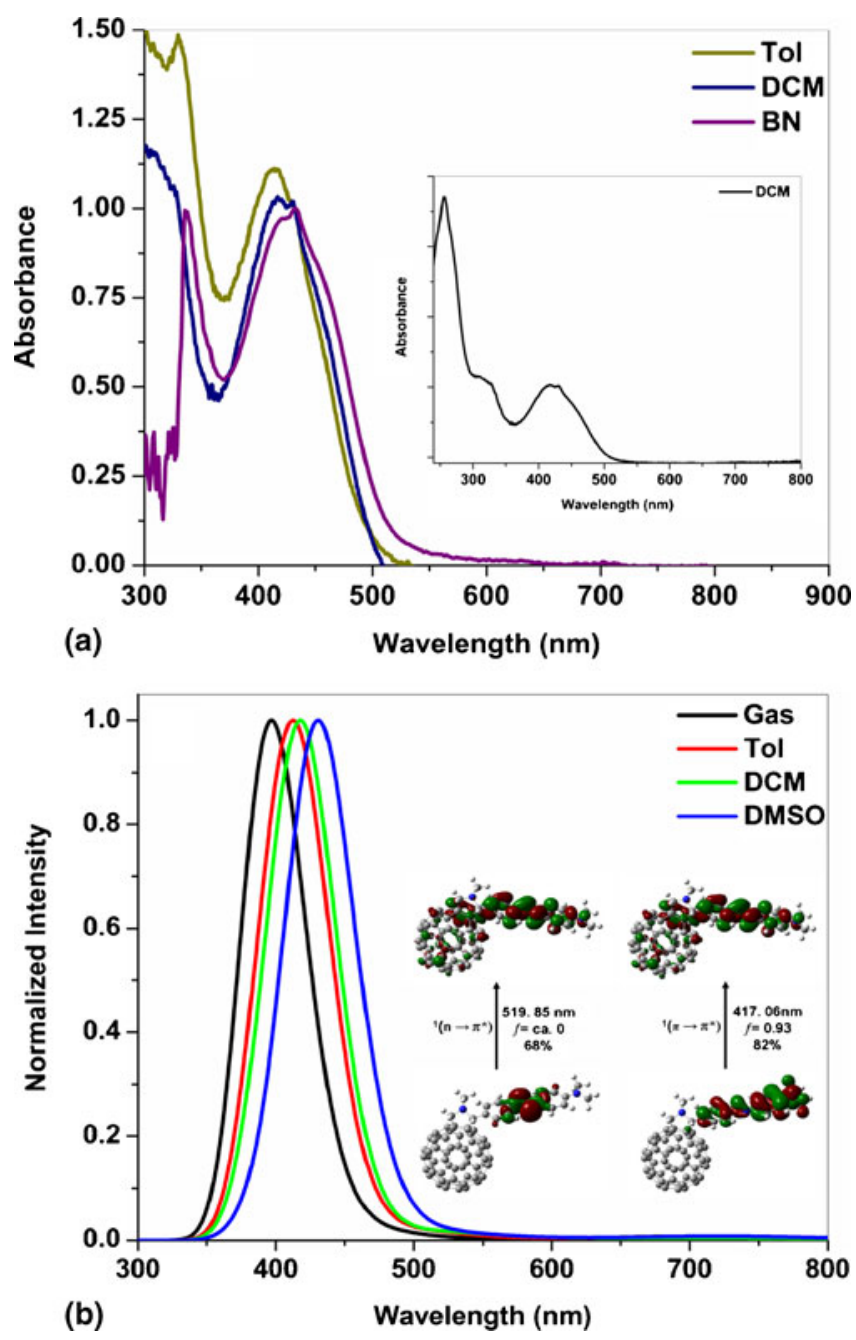

Figure 4. (a) UV-Vis absorption spectra of $1 \times 10^{-5}$ DPNME in toluene, dichloromethane and benzonitrile solvents; inset shows the full spectrum of DPNME in dichloromethane (DCM) solvent. (b) TD - B3LYP/ 3 21G(d) // B3LYP/ 6-31G(d, p) calculated absorption spectra of DPNME in toluene, dichloromethane and benzonitrile solvents; inset shows the molecular orbitals involved in the electronic transitions ${ }^{1}\left(\mathrm{n} \rightarrow \pi^{*}\right)$ and ${ }^{1}(\pi \rightarrow \pi)^{*}$ of DPNME. 

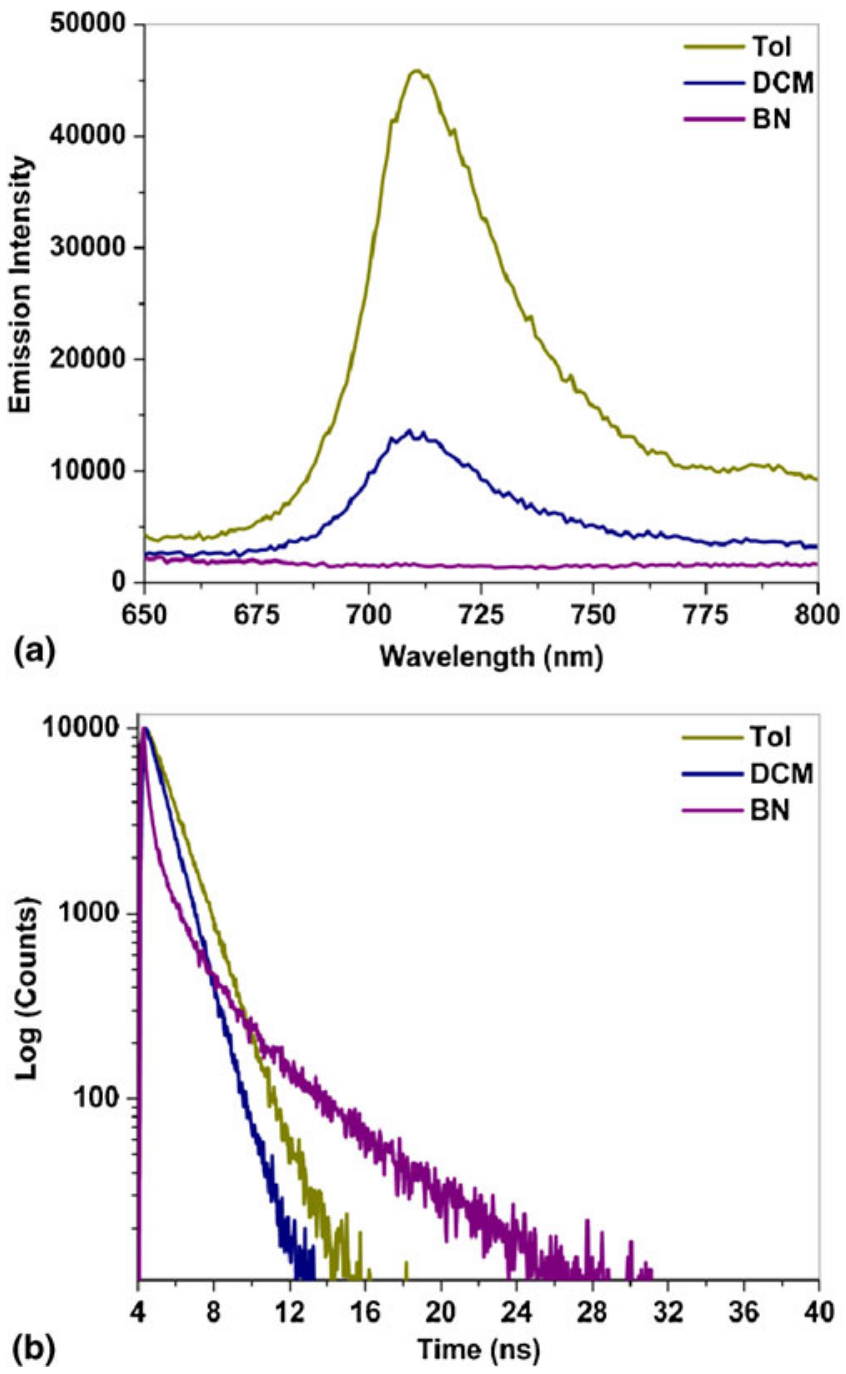

Figure 5. (a) Steady state emission spectra and (b) fluorescence life time plots of DPNME in toluene, dichloromethane and benzonitrile solvents.

polar solvent. The TD-DFT predicted electronic transition energies in vacuum and solvent media were found to be in excellent agreement with the experimentally obtained values, shown in figure $4 \mathrm{~b}$. Analysis of the MO coefficients involved in the ${ }^{1}\left(\pi \rightarrow \pi^{*}\right)$ and ${ }^{1}\left(\mathrm{n} \rightarrow \pi^{*}\right)$ electronic transitions predicted at 519.85 and $417.06 \mathrm{~nm}$ in vacuum revealed slight charge transfer character as evidenced from the molecular orbital picture, collected in the inset of figure $4 \mathrm{~b}$, wherein electronic excitation involves electron transition from orbitals completely localized on the donor to orbitals delocalized over both donor and the acceptor. No change in the molecular orbital ordering and nature of MO coefficients apart from slight stabilization in MO energies was observed while traversing from vacuum to solvent media of varying polarity. Hence, the vacuum calculated molecular orbital composition for ${ }^{1}\left(\pi \rightarrow \pi^{*}\right)$ and ${ }^{1}\left(\mathrm{n} \rightarrow \pi^{*}\right)$ electronic excitation processes could also be applicable in solvent media.

\subsection{Excited state electron transfer dynamics in DPNME dyad}

To get insight into the excited state properties of the DPNME dyad, steady state fluorescence spectra were acquired in toluene, dichloromethane and benzonitrile, as depicted in figure $5 \mathrm{a}$. The emission band at $713 \mathrm{~nm}$ upon excitation at $430 \mathrm{~nm}$ decreased in intensity upon increase in solvent polarity from toluene to dichloromethane and was completely quenched in the more polar benzonitrile.

Time resolved fluorescence life time studies revealed a single exponential decay pattern for DPNME in toluene and dichloromethane with a reduced life time in dichloromethane (cf. figure 5b), whereas in benzonitrile, the decay was tri-exponential in nature with the lowest life time component having a larger contribution. A reduced life time of DPNME in more polar solvents was in accordance with the steady state fluorescence emission behaviour. From the life time of the reference compound $N$-methylfulleropyrrolidine (NMFP), the rate and quantum yield for intramolecular charge separation were calculated for DPNME in dichloromethane and benzonitrile adapting the established equations 1 and 2 as follows:

$$
\begin{gathered}
\mathrm{k}_{\mathrm{cs}}=1 / \tau-1 / \tau_{\mathrm{ref}} . \\
\left(\tau_{\mathrm{ref}}=1.21 \mathrm{~ns}\right) \\
\Phi_{c s}=\left[\mathrm{k}_{\mathrm{cs}} /\left[1 / \tau_{\mathrm{ref}}+\mathrm{k}_{\mathrm{cs}}\right]\right] \times 100 .
\end{gathered}
$$

Table 2. Quantum yield of fluorescence $\left(\Phi_{\mathrm{f}}\right)$, fluorescence life time $(\tau)$, rate of charge separation $\left(\mathrm{k}_{\mathrm{cs}}\right)$ and quantum yield $\left(\Phi_{\mathrm{cs}}\right)$ of charge separation in toluene, dichloromethane and benzonitrile.

\begin{tabular}{lclcc}
\hline Solvent $(\varepsilon)$ & $\Phi_{\mathrm{f}}\left(\times 10^{4}\right)$ & $\tau(\mathrm{ns})$ & $\mathrm{k}_{\mathrm{cs}}\left(\mathrm{s}^{-1}\right)$ & $\Phi_{\mathrm{cs}}$ \\
\hline Toluene (2.38) & 10 & 1.41 & - & - \\
DCM (8.93) & 5.59 & 1.06 & $0.124 \times 10^{9}$ & $0.1318(13.18 \%)$ \\
BN (25.2) & 0.127 & 0.196 & $4.29 \times 10^{9}$ & $0.84(84 \%)$ \\
\hline
\end{tabular}



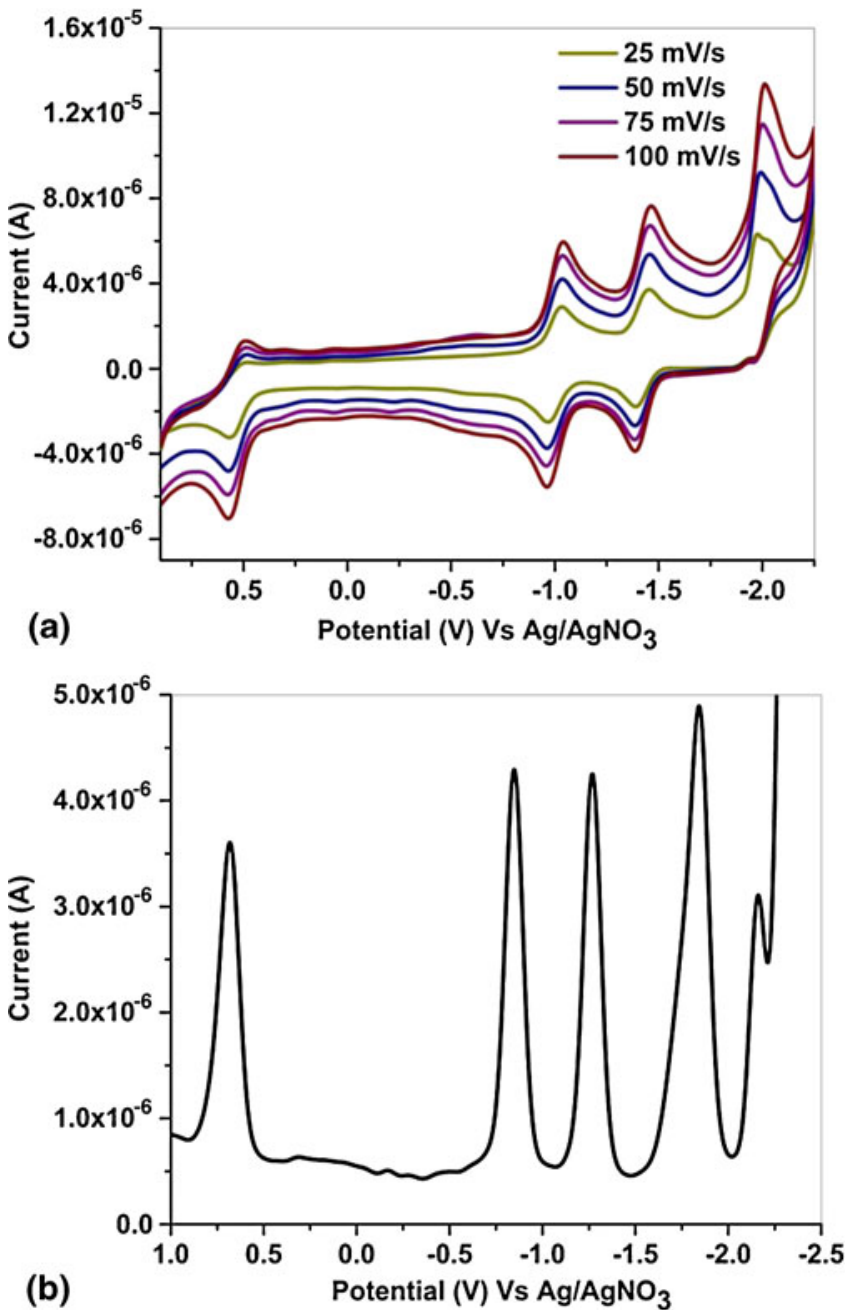

Figure 6. (a) Cyclic voltammogram and (b) DPV of DPNME dyad in benzonitrile solvent (supporting electrolyte: TBAF, reference electrode: $\mathrm{Ag} / \mathrm{AgNO}_{3}$, working electrode: glassy carbon, counter electrode: Pt wire).

A similar calculation in toluene was not feasible due to larger fluorescence life times obtained for DPNME than the NMFP reference. Increased rate constants and quantum yields for charge separation resulted upon moving from less polar dichloromethane to a more polar benzonitrile; notably $84 \%$ of the singlet excited state of the DPNME was converted into a charge separated state in benzonitrile, vide table 2 .

Keeping abreast of the facts discussed, electrochemical experiments in benzonitrile (cf. figure 6a, b) probed energetics of the electron transfer process; the first oxidation and reduction potentials in the differential pulse voltammogram (DPV) shown in figure 6b provided energy of the charge separated state in benzonitrile. Similar data in toluene and dichloromethane, for which electrochemical data were unavailable, were estimated by using the Weller equation ${ }^{42}$ (vide equation 3 ) and are shown in table 3. Dielectric constants of Tol and DCM were used separately to calculate the free energy change by taking benzonitrile as a reference as in eq. 3 , i.e. the $\varepsilon_{\mathrm{s}}$ and $\varepsilon_{\text {ref }}$ terms.

$$
\begin{aligned}
\Delta G_{\mathrm{cs}}= & e\left(E^{\mathrm{ox}}(D)-E^{\mathrm{red}}(A)\right)-E_{00} \\
& -e^{2} / 4 \pi \varepsilon_{\mathrm{o}} \varepsilon_{\mathrm{s}} R_{\mathrm{cc}} \\
& -e^{2} / 8 \pi \varepsilon_{o}\left(1 / r^{+}+1 / r^{-}\right)\left(1 / \varepsilon_{\mathrm{ref}}-1 / \varepsilon_{\mathrm{s}}\right),
\end{aligned}
$$

where

$E^{\text {ox }}(D)=$ oxidation potential of the donor $(0.5319 \mathrm{~V})$

$E^{\text {red }}(A)=$ reduction potential of the acceptor $(-1.001 \mathrm{~V})$

$E_{00}=$ singlet or triplet state energy $(1.72 \mathrm{eV}$ or $1.5 \mathrm{eV}$ )

$R_{\mathrm{cc}}=$ centre to centre distance $(1.18 \mathrm{~nm})$,

$r^{+}=$effective ionic radii of the donor

$(0.37 \mathrm{~nm})$, and

$r^{-}=$effective ionic radii of the acceptor

$(0.56 \mathrm{~nm})$.

Centre to centre distance, radius of the donor and radius of the acceptor were obtained from DFT calculations, $\mathrm{E}_{00}$ value of $1.72 \mathrm{eV}$ and $1.50 \mathrm{eV}$ were used for fullerene singlet and triplet states, respectively. ${ }^{43}$ As for the photo-induced process arising from the lowest fullerene singlet state, it was seen that the activation energy barrier is higher compared to the free energy of charge separation in toluene, explaining the absence of quenching in the solvent. For benzonitrile,

Table 3. Reorganization energy $(\lambda)$, free energy change $\left(\Delta \mathrm{G}_{\mathrm{CS}}\right)$, and the activation energy barrier $\left(\Delta \mathrm{G}_{\mathrm{CS}}^{\#}\right)$ for charge separation in toluene, dichloromethane and benzonitrile.

\begin{tabular}{lcccccc}
\hline & \multicolumn{3}{c}{${ }^{1} \mathrm{C}_{60} *-$ Donor } & & \multicolumn{2}{c}{${ }^{3} \mathrm{C}_{60} *-$ Donor } \\
\cline { 2 - 3 } \cline { 6 - 7 } Solvent & $\lambda(\mathrm{eV})$ & $\Delta \mathrm{G}_{\mathrm{cs}}(\mathrm{eV})$ & $\Delta \mathrm{G}_{\mathrm{cs}}^{\#}(\mathrm{eV})$ & & $\Delta \mathrm{G}_{\mathrm{cs}}(\mathrm{eV})$ & $\Delta \mathrm{G}_{\mathrm{cs}}^{\#}(\mathrm{eV})$ \\
\hline Toluene & 0.33 & 0.51 & 0.53 & & 0.76 & 0.89 \\
DCM & 0.81 & -0.02 & 0.19 & & 0.2 & 0.31 \\
BN & 0.82 & -0.24 & 0.10 & & -0.016 & 0.2 \\
\hline
\end{tabular}


the free energy of charge separation was more negative than the activation barrier allowing electron transfer from donor to the acceptor and quenching of the excited singlet state, as was evident from the decreased fluorescence intensity in figure 5a. Charge separation from the fullerene triplet excited state was ruled out in toluene and dichloromethane solvents while a meager possibility of it in the more polar benzonitrile solvent was evidenced from the parameters listed in table 2 . The above discussions unambiguously proved electron transfer to have quenched the fullerene singlet excited state emission in polar benzonitrile solvent.

The other important facet of electron transfer was the reorganization energy associated with the DPNME dyadic system. The calculated total reorganization energy of $0.33 \mathrm{eV}$ in toluene solvent was very close to the internal reorganization energy of fullerene $(0.3 \mathrm{eV})^{2}$, implying a non-existent electron transfer event in toluene. The former is defined as,

$$
\lambda=\lambda_{\mathrm{s}}+\lambda_{\mathrm{i}},
$$

with $\lambda_{\mathrm{s}}$ as the solvent reorganization term (vide eqn. 5) and $\lambda_{\mathrm{i}}$, the internal reorganization energy, set at $0.3 \mathrm{eV}$.

$$
\begin{gathered}
\lambda_{s}=e^{2} / 4 \pi \varepsilon_{0}\left[1 / 2\left[1 / r_{+}+1 / r_{-}\right]-1 / R_{\mathrm{cc}}\right]\left[1 / n^{2}-1 / \varepsilon_{s}\right] \\
\Delta G_{\mathrm{cs}}^{\#}=\left(\Delta G_{\mathrm{cs}}+\lambda\right)^{2} / 4 \lambda^{2} .
\end{gathered}
$$

The situation is interesting in dichloromethane and benzonitrile solvents. The higher magnitude of total reorganization energy is in excellent corroboration with the steady state and time resolved fluorescence experiments, showcasing charge separation. Further, the near similar magnitude of total reorganization energy in dichloromethane and benzonitrile solvents revealed very small structural reorganization associated with the 3D fullerene moiety upon charge separation, justifying its role as an efficient acceptor in comparison with other 2D molecular systems. ${ }^{44}$

\subsection{Electron transfer kinetics of DPNME at the electrode/electrolyte interface}

The electrochemistry of fullerene $\mathrm{C}_{60}$ is of interest as it provides information on the associated energetics and kinetics of electron transfer processes and chemical reactions at an electrode/electrolyte interface. Studies on the reduction of $\mathrm{C}_{60}$ in solution phase has produced a series of diffusion controlled Nernstian reduction waves, showing successive addition of electrons to the molecule, according to equation 7 .

$$
C_{60}^{\mathrm{n}-}+\mathrm{e}^{-} \leftrightarrow C_{60}^{(\mathrm{n}+1)-} ; \mathrm{n}=0,1,2, \ldots ., 5 .
$$

In line with the reduction process in pristine $\mathrm{C}_{60}$, functionalized $\mathrm{C}_{60}$ or $\mathrm{C}_{60}$ derivatives were also reported to retain the electrochemical properties with cathodically shifted reduction potentials due to the loss of degeneracy of the LUMOs and thus with reduced $\pi$ character. Reports on the interfacial electron transfer across electrode/electrolyte interfaces predicted reversible and quasi-reversible electron transfer kinetics for freely diffusing $\mathrm{C}_{60}$ derivatives under study. ${ }^{45,46}$ For a nonNernstian multi-step process deviated from equilibrium, the details of its kinetics influenced its electrochemical behaviour. With quantification of the kinetic parameters, the mechanism of the redox process could be diagnosed. For such quasi-reversible systems, the diffusion current $I_{d}$ was controlled by both mass transfer
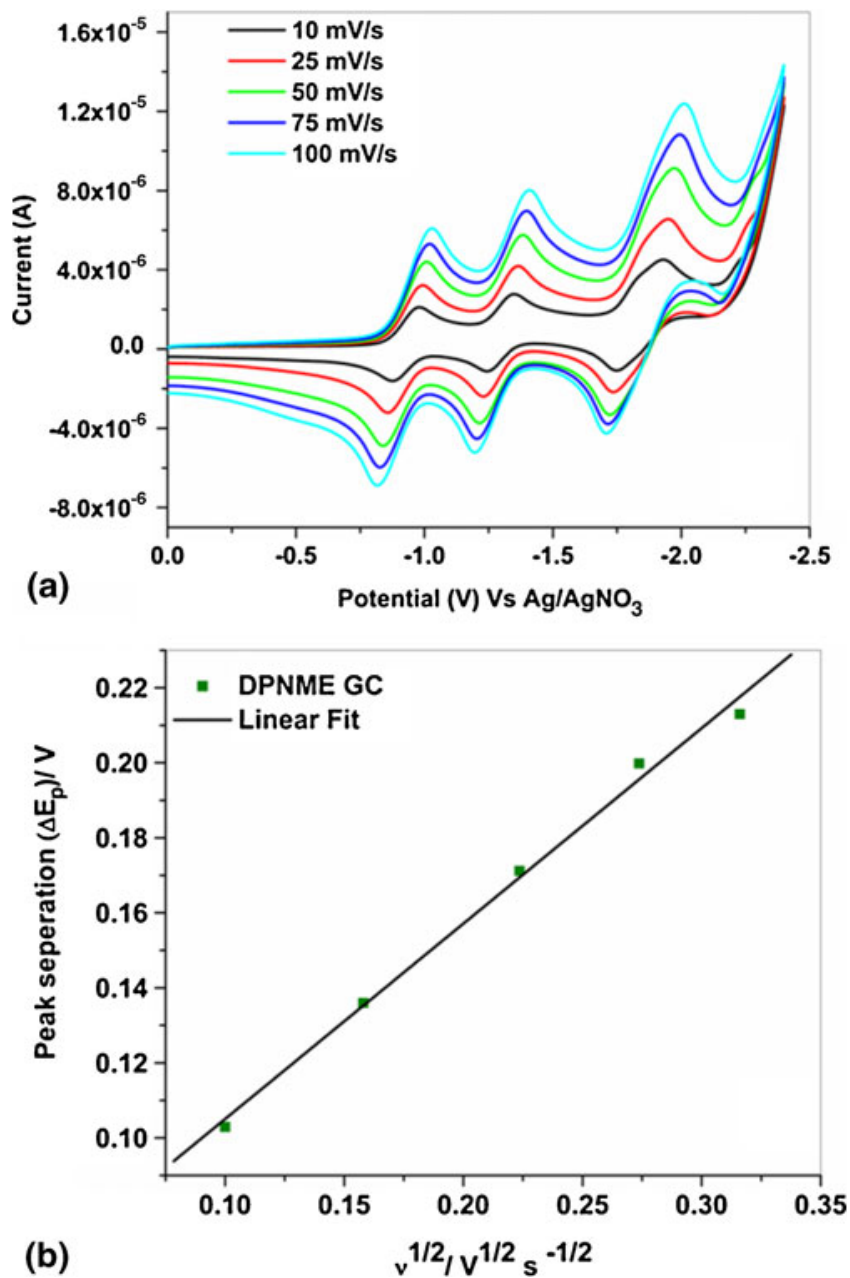

Figure 7. (a) Cyclic voltammograms of $5 \times 10^{-4} \mathrm{M}$ DPNME at varying scan rates $\left(\mathrm{V} . \mathrm{s}^{-1}\right.$ ) at a glassy carbon electrode [Solvent: benzonitrile; Supporting electrolyte: $0.1 \mathrm{M}$ TBAF; Reference electrode: $\mathrm{Ag} / \mathrm{AgNO}_{3}$; Counter electrode: $\mathrm{Pt}$ ]. (b) Scan rate dependence showing the plot of $\Delta E_{P} v s v^{1 / 2}$ for the DPNME/ DPNME ${ }^{1-}$ solution phase redox couple. 
and heterogeneous electron transfer routes. ${ }^{47}$ With GC and $\mathrm{Pt}$ working electrodes of radii $1.5 \mathrm{~mm}$, the kinetics of electron transfer associated with equation 8 , across the electrolyte/electrode surface was investigated from the cyclic voltammograms of DPNME acquired in 4:1 toluene/acetonitrile solvent.

$$
\mathrm{DPNME}+\mathrm{e}^{-} \longrightarrow \mathrm{DPNME}^{1-} \text {. }
$$

All the cyclic voltammograms were recorded at a constant temperature of $25^{\circ} \mathrm{C}$. The anodic to cathodic current ratio, $\mathrm{I}_{\mathrm{pa}} / \mathrm{I}_{\mathrm{pc}}$ for DPNME/DPNME ${ }^{1-}$ couple was measured for each cyclic voltammogram, varying the scan rate from 0.01 up to $0.1 \mathrm{Vs}^{-1}$. The standard heterogeneous charge transfer rate constant $\left(\mathrm{k}_{\text {het }}\right)$ associated with the redox process was calculated from the peakto-peak separation $\left(\Delta \mathrm{E}_{\mathrm{P}}\right)$, according to Nicholson. ${ }^{48}$ Figures $7 \mathrm{a}$ and $8 \mathrm{a}$ contain the quasi-reversible electron transfer processes at the electrode/solution interface as

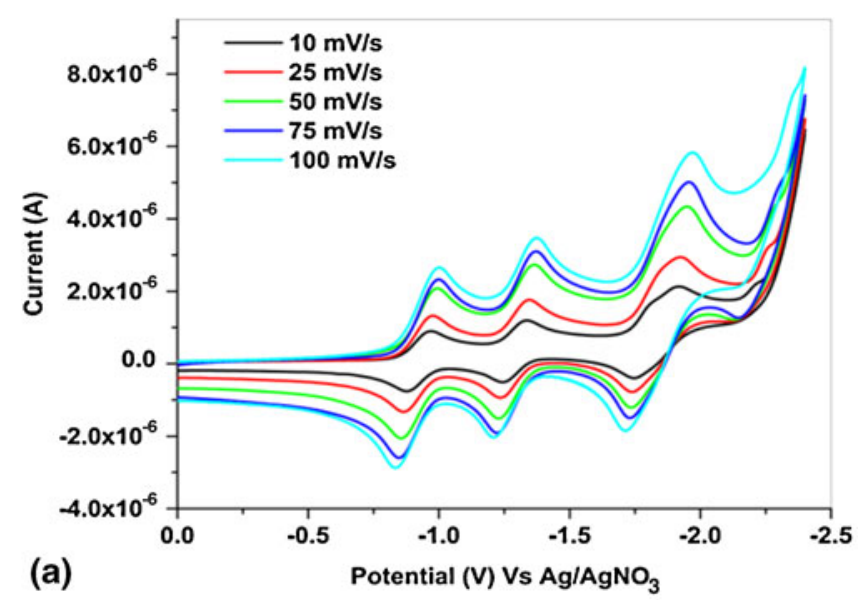

(a) Potential (V) Vs Ag/AgNO

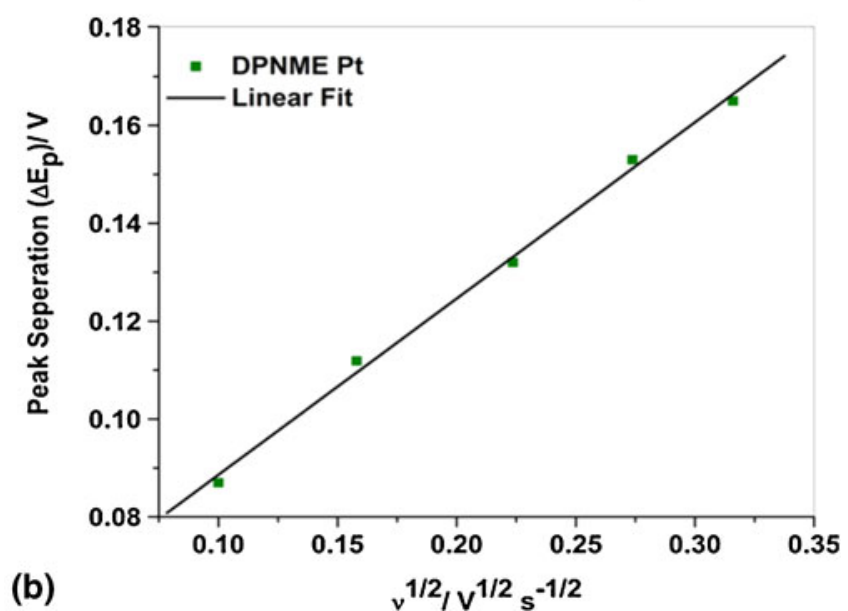

Figure 8. (a) Cyclic voltammograms of $5 \times 10^{-4} \mathrm{M}$ DPNME at varying scan rates $\left(\mathrm{V} . \mathrm{s}^{-1}\right)$ at a platinum electrode [Solvent: benzonitrile; Supporting electrolyte: 0.1 M TBAF; Reference electrode: $\mathrm{Ag} / \mathrm{AgNO}_{3}$; Counter electrode: $\left.\mathrm{Pt}\right]$. (b) Plot of $\Delta \mathrm{E}_{\mathrm{P}}$ vs $v^{1 / 2}$ for the DPNME/ DPNME ${ }^{1-}$ solution phase redox process.

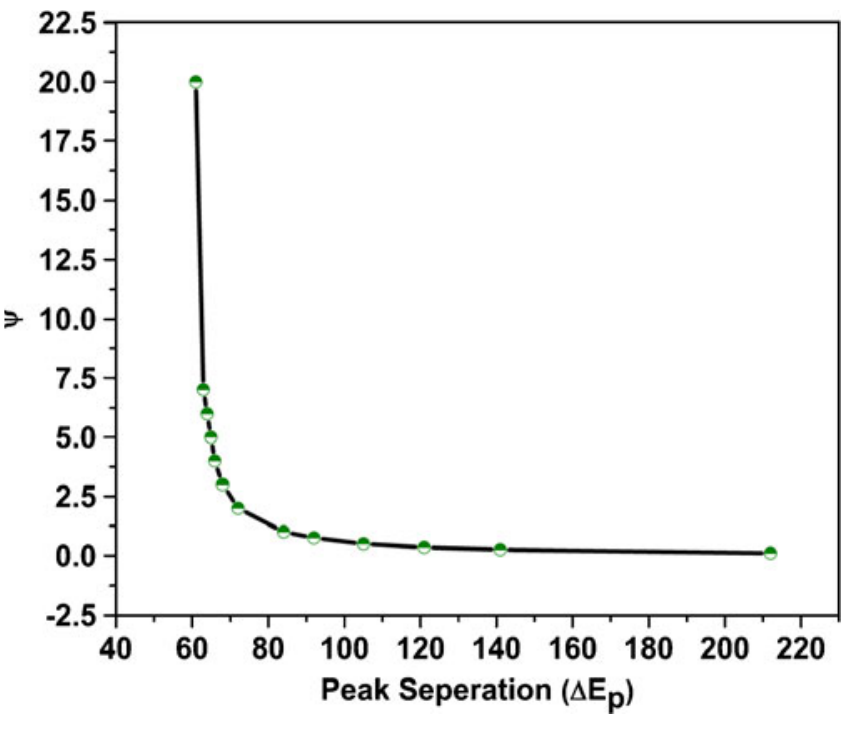

Figure 9. Plot of $\psi$ vs $\Delta \mathrm{E}_{\mathrm{P}}$ for a quasi reversible reaction, drawn in reference to the reported data according to Bard and Faulkner. ${ }^{47}$

a function of working electrode type. The plots of $\Delta E_{P}$ vs $v^{1 / 2}$ as shown in figures $7 \mathrm{~b}$ and $8 \mathrm{~b}$ yielded a linear behaviour. The very close proximity of $\mathrm{I}_{\mathrm{pa}} / \mathrm{I}_{\mathrm{pc}} \sim 1$ indicated $\alpha$ to be 0.5 for the aforementioned quasi reversible processes.

Under these conditions of quasi-reversibility, it was possible to study kinetics of the electrode reaction where separation of the peak potentials, $\Delta \mathrm{E}_{\mathrm{P}}$, was the measure of the standard rate constant for electron transfer. These $\Delta E_{p}$ values were introduced in the working curve described by Nicholson ${ }^{48}$ for obtaining the quasi-reversible current function (transfer parameter), $\psi$ (vide figure 9), from which the standard heterogeneous charge transfer rate constant for electron transfer was calculated according to equation 9 ,

$$
k_{\text {het }}=\psi[D \pi v(n F / R T)]^{1 / 2},
$$

where $v$ is the scan rate, $\mathrm{F}$, the Faraday constant and $\mathrm{D}$ is the diffusion coefficient. From the $\psi$ vs. $\Delta \mathrm{E}_{\mathrm{P}}$

Table 4. Heterogeneous rate constants $\left(\mathrm{k}_{\text {het }}\right)$ associated with the DPNME/DPNME ${ }^{1-}$ redox couple at the glassy carbon and platinum electrode/electrolyte interfaces.

\begin{tabular}{lcc}
\hline Scan rate & \multicolumn{2}{c}{$\mathrm{k}_{\text {het }} \times 10^{3}\left(\mathrm{~cm} . \mathrm{s}^{-1}\right)$} \\
\cline { 2 - 3 }$v\left(\mathrm{~V}^{-1}\right)$ & Glassy carbon & Platinum \\
\hline 0.01 & 1.52 & 2.59 \\
0.025 & 1.59 & 2.02 \\
0.05 & 1.64 & 2.19 \\
0.075 & 1.29 & 1.95 \\
0.1 & 1.49 & 2.26 \\
\hline
\end{tabular}


curve, the corresponding $\psi$ values related to $\Delta \mathrm{E}_{\mathrm{P}}$ could be obtained for calculation of $k_{\text {het }}$ for each scan rate. The overall $k_{\text {het }}$ was deduced by averaging the $k_{\text {het }}$ values obtained for individual scan rates and are tabulated in table 4 for glassy carbon and platinum electrodes; the heterogeneous electron transfer rate constants at the platinum electrode surfaces revealed a faster kinetics. The values were in good agreement with those for typical quasi-reversible reactions ranging between $10^{-1}$ and $10^{-5} \mathrm{~cm} \mathrm{~s}^{-1} .49$

\section{Conclusions}

Facile photo-induced intramolecular electron transfer was observed in the fullerene - N,N - dimethylaminoazobenzene based dyad system. The DFT calculated ground state electronic structure provided the frontier molecular orbital characteristics sufficing the essentials for the hybrid molecular skeleton to be used as a futuristic molecular device. TD-DFT modelling of the UV-Vis spectra of the dyad in solvents of varying polarity showed mixing of charge transfer excitons with ${ }^{1}\left(\mathrm{n} \rightarrow \pi^{*}\right)$ and ${ }^{1}(\pi \rightarrow \pi)^{*}$ electronic transitions involved with the azo chromophore, justifying the experimentally observed bathochromic shifting of the absorption maxima as a function of solvent polarity. Quenched fluorescence and reduced life times in more polar benzonitrile solvent testified deactivation of the singlet excited state of the dyad via charge separation. The important parameters involved in the electron transfer event were estimated adapting Weller, Marcus and Born-Hush approximations.

The obtained kinetic and thermodynamic parameters for the intramolecular charge separation in the dyad were in excellent similitude with the macroscopic donor-bridge-acceptor systems constituting donor moieties, such as, porphyrins and pthalocyanins, implying its structural prominence. Unlike the other reported systems, the incorporated photo tunable/conformationally flexible azo bridge between the $\mathrm{N}, \mathrm{N}$-dimethylaniline donor and the fullerene acceptor in the dyad will shorten the intramolecular donor acceptor distance upon light induced trans $\rightarrow$ cis isomerization enabling more efficient charge separation, as testified from DFT calculations. In addition, in this conformation, the curvature of $\mathrm{C}_{60}$ will allow its $\pi$ orbitals to orient isotropically for a maximum intermolecular overlap towards an ideal organic conductor. The estimated heterogeneous electron transfer rate constants for the trans-dyad revealed a quasi-reversible electron transfer pathway for the dyad/dyad ${ }^{1-}$ couple across the electrode/electrolyte interface.

\section{Acknowledgements}

The authors thank the Department of Science and Technology (DST), New Delhi for the financial support (Grant No. SR/S2/CMP-57/2006) and Prof. P Ramamurthy, National centre for Ultrafast Processes, University of Madras for life time measurements.

\section{References}

1. Metzer R M 2003 Chem. Rev. 1033803

2. Guldi D M 2000 Chem. Commun. 321

3. Williams R M, Zwier J M and Verhoeven J M $1995 \mathrm{~J}$. Am. Chem. Soc. 1174093

4. Arakia Y and Itoa O 2008 J. Photochem. Photobiol. C: Photochem. Rev. 993

5. Gayathri S S and Patnaik A 2006 Chem. Commun. 1977

6. Prato M 1997 J. Mater. Chem. 71097

7. Kadish K M and Ruoff R M Eds. 2000 Fullerenes: Chemistry, physics and technology (New York: WileyInterscience)

8. Figueira-Duarte T M, Gegout A and Nierengarten J F 2007 Chem. Commun. 109

9. Thomas K G, Biju V, Guldi D M, Kamat P V and George M V 1999 J. Phys. Chem. A 103(50) 10755

10. Flint D G, Kumita J R, Smart O S and Woolley G A 2002 Chem. Biol. 939

11. Ikeda T and Tsutsumi O 1995 Science 2681873

12. Liu Z F, Hashimoto K and Fujishima K 1990 Nature 347 658

13. Kawata S and Kawata Y 2000 Chem. Rev. 1001777

14. Astrand P O, Ramanujam P S, Hvilsted S, Bak K L and Sauer Stephan P A 2000 J. Am. Chem. Soc. 1223482

15. Kay K Y, Han K J, Yu Y J and Park Y D 2002 Tetrahedron Lett. 435053

16. Oh-ishi K, Okamura J, Ishi-I T, Sano M and Shinkai S 1999 Langmuir 152224

17. Shirai Y, Sasaki T, Guerrero J M, Byung-Chan Y, Hodge $\mathrm{P}$ and Tour J M 2008 ACS Nano 2(1) 97

18. Schuster D I, Li K, Guldi D M, Palkar A, Echegoyen L, Stanisky C, Cross R J, Niemi M, Tkachenko N V and Lemmetyinen H 2007 J. Am. Chem. Soc. 12915973

19. Armaroli N, Marconi G, Echegoyen L, Bourgeois J P and Diederich F 2000 Chem-Eur. J. 61629

20. Armaroli N, Accorsi G, Song F, Palkar A, Echegoyen L, Bonifazi D and Diederich F 2005 Chem. Phys. Chem. 6(4) 732

21. Cramariuc O, Hukka T I, Rantala T T, and Lemmetyinen H 2006 J. Phys. Chem. A. 110(45) 12470

22. D'Souza F, Chitta R, Sandanayaka A S D, Subbaiyan N K, D'Souza L, Araki Y and Ito O 2007 J. Am. Chem. Soc. 129(51) 15865

23. Shen Y, Reparaz J S, Wagner M R, Hoffmann A, Thomsen C, Lee J O, Heeg S, Hatting B, Reich S, Saeki A, Seki S, Yoshida K, Babu S S, Möhwald H and Nakanishi T 2011 Chem. Sci. 22243

24. Balzani V, Credi A and Venturi M 2008 Chem. Sus. Chem. 126

25. Kumar K S and Patnaik A 2010 J. Comput. Chem. 361182

26. Kumar K S and Patnaik A 2010 Chem. Phys. Chem. 11 3645 
27. Kumar K S and Patnaik A 2011 Chem-Eur. J. 175327

28. Kumar K S and Patnaik A 2011 Langmuir 27(17) 11017

29. Frisch M J et al. 2004 Gaussian 03, Revision C.02, Gaussian, Inc., Wallingford CT

30. Lee C, Yang W and Parr R G 1988 Phys. Rev. B 37785

31. Becke A D 1988 Phy. Rev. A. 383098

32. Vosko S H, Wilk L and Nusair M 1980 Can. J. Phys. 58 1200

33. Stephens P J, Devlin F J, Chabalowski C F and Frisch M J 1994 J. Phys. Chem. 9811623

34. Casida M 1995 Recent advances in density functional methods (ed) D P Chong (Singapore: World Scientific) Vol. 1

35. Casida M 1996 Recent developments and applications of modern density functional theory (ed) J M Seminario (Amsterdam: Elsevier)

36. Amovilli C, Barone V, Cammi R, Cance E, Cossi M, Mennucci B, Pomelli C S and Tomasi 1998 J. Adv. Quantum Chem. 32227

37. Cossi M and Barone V 2001 J. Chem. Phys. 1154708

38. Aviram A and Ratner M A 1974 Chem. Phy. Lett. 29(2) 277
39. Mujicaa V, Ratner M A and Nitzan A 2002 Chem. Phys. 281147

40. Majumder C, Mizuseki H and Kawazoe Y 2001 Phys. Chem. A 105(41) 9454

41. Maggini M, Scorrano G and Prato M 1993 J. Am. Chem. Soc. 1159798

42. Rehm D and Weller A 1970 Isr. J. Chem. 8259

43. Accorsi G and Armaroli N 2010 J. Phys. Chem. C 114(3) 1385

44. Imahori H, Hagiwara K, Akiyama T, Aoki M, Taniguchi S, Okada T, Shirakawa M and Sakata Y 1996 Chem. Phys. Lett. 263545

45. Martin N, Sanchez L, Illescas B and Perez I 1998 Chem. Rev. 98(7) 2527

46. Langa F, de la Cruz P, Espildpra E, Gonzalez-Cortes, de la Hoz A and Lopez-Arza V 2000 J. Org. Chem. 65(25) 8675

47. Bard A J and Faulkner L R 2000 Electrochemical methods fundamentals and applications (John Wiley \& sons)

48. Nicholson R S 1965 Anal. Chem. 37(11) 1351

49. Wang J 2000 Analytical electrochemistry 2nd edition (WILEY-VCH) p. 33 